Supplement of Nat. Hazards Earth Syst. Sci., 18, 2877-2891, 2018

https://doi.org/10.5194/nhess-18-2877-2018-supplement

(C) Author(s) 2018. This work is distributed under

the Creative Commons Attribution 4.0 License.

(c) (1)

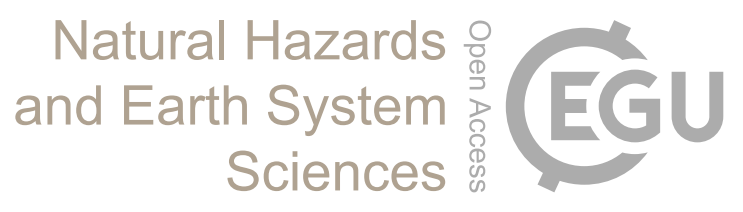

Supplement of

\title{
Evaluating critical rainfall conditions for large-scale landslides by detecting event times from seismic records
}

\section{Hsien-Li Kuo et al.}

Correspondence to: Guan-Wei Lin (gwlin@mail.ncku.edu.tw)

The copyright of individual parts of the supplement might differ from the CC BY 4.0 License. 


\section{S1. List of rainfall events chosen in the study}

In the study, totally nineteen rainfall events including seventeen typhoons and two heavy rainfall events occurring in the period of 2005-2014 were chosen to examine the seismic records and identify landslide-induced signals (Table S1).

Table S1. Heavy Rainfall Event List

\begin{tabular}{lll}
\hline & Event & Date (year/month/date) \\
\hline 1 & Haitang & $2005 / 07 / 16-07 / 20$ \\
2 & Talim & $2005 / 08 / 30-09 / 01$ \\
3 & 0609 Rain & $2005 / 06 / 09$ \\
4 & Bilis & $2005 / 07 / 12-07 / 15$ \\
5 & 0604 Rain & $2006 / 06 / 04$ \\
6 & Kalmaegi & $2006 / 07 / 16-07 / 18$ \\
7 & Fung-Wong & $2008 / 07 / 26-07 / 29$ \\
8 & Sinlaku & $2008 / 09 / 11-09 / 16$ \\
9 & Morakot & $2009 / 08 / 05-08 / 10$ \\
10 & Fanapi & $2010 / 09 / 17-09 / 20$ \\
11 & Megi & $2010 / 10 / 21-10 / 23$ \\
12 & Nanmadol & $2011 / 08 / 27-08 / 31$ \\
13 & Talim & $2012 / 06 / 19-06 / 21$ \\
14 & Saola & $2012 / 07 / 31-08 / 03$ \\
15 & Tembin & $2012 / 08 / 21-08 / 25$ \\
16 & Soulik & $2013 / 07 / 11-07 / 13$ \\
17 & Trami & $2013 / 08 / 20-08 / 22$ \\
18 & Matmo & $2014 / 07 / 21-07 / 23$ \\
19 & Fung-Wong & $2014 / 09 / 19-09 / 22$ \\
\hline
\end{tabular}


S2. Waveforms and spectrograms of the seismic signals induced by ID 1 landslide that occurred in 2005

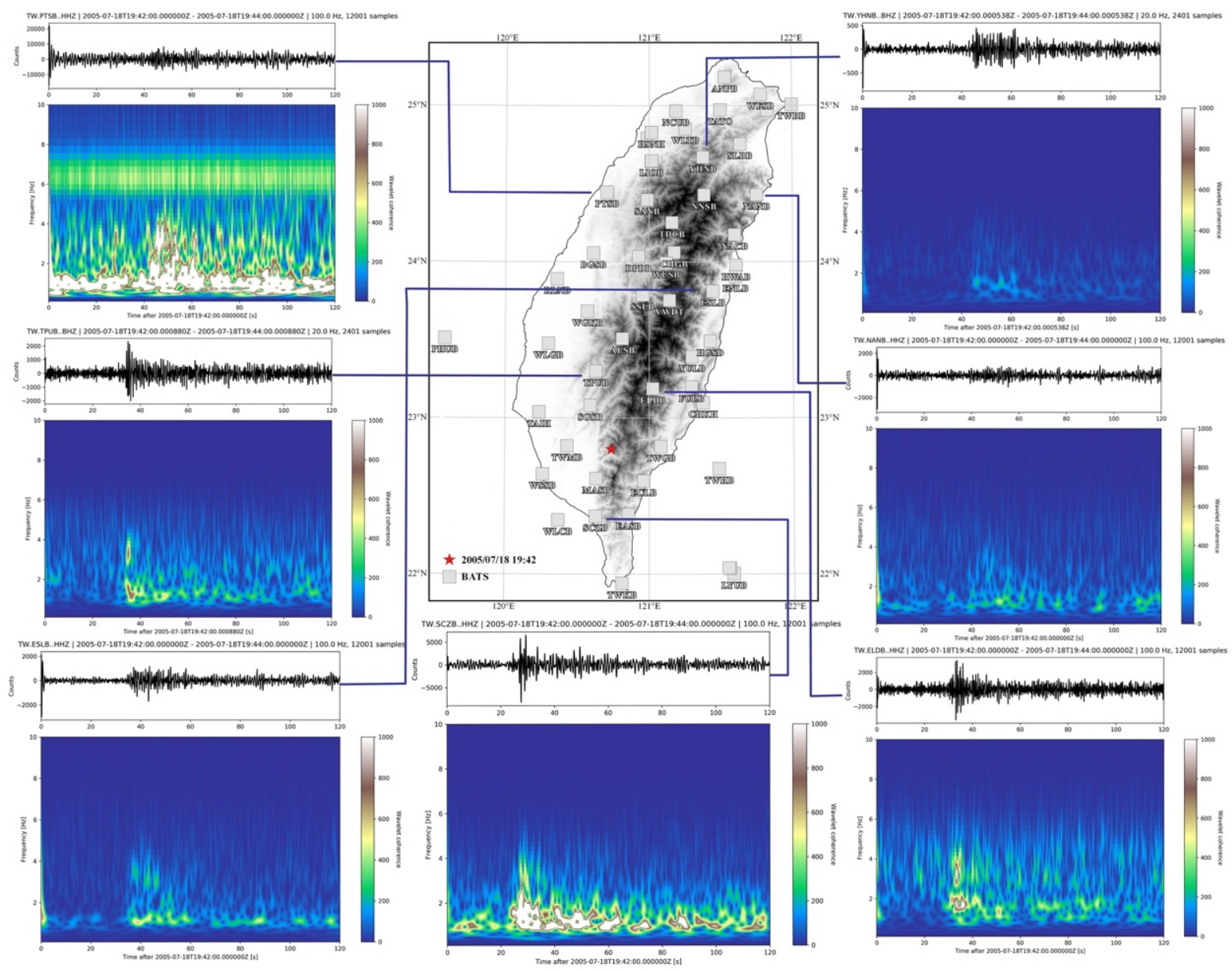

Fig S1. A sequence of original waveforms and spectrograms of the vertical-component signals induced by ID 1 landslide that occurred in 2005. 


\section{S3. Validation of the selection method for rainfall stations}

The effect of rain gauge distribution over the accuracy of rainfall has been assessed using gauge observation in a $35 \mathrm{~km} \times 50 \mathrm{~km}$ region of south Taiwan (Fig. S1). The amounts of daily rainfall during 2009 Typhoon Morakot (8/6-8/11) recorded at 19 rain gauge stations were selected to validate the accuracy of rainfall. At first, the amounts of daily rainfall were interpolated to $01 \mathrm{~V} 040$ station using IDW methods. The errors between measurements and interpolated data were smaller than $15 \%$. It indicates IDW method can be used to interpolate rainfall to a selected location in our study area. Secondly, the amounts of daily rainfall at the central point of the $35 \mathrm{~km} \times 50 \mathrm{~km}$ region were estimated. The errors of daily rainfall between the central point and the nearest rain gauge station (01V040) were smaller than $10 \%(0.5 \%-10 \%$ at different date). Besides, the correlation coefficients would keep at $90 \%$ as a distance between the central point and rain gauge stations less than $20 \mathrm{~km}$, and even keep at $98 \%$ as a distance less than $10 \mathrm{~km}$ (Fig. S2). Therefore, in the study, an upper limit of basin area smaller than $100 \mathrm{~km} 2(10 \mathrm{~km} \times 10 \mathrm{~km}$ was adopted to avoid a significant decrease of the accuracy of rainfall.

The influence of topography on rainfall variability has been analyzed in the same 35 $\mathrm{km} \times 50 \mathrm{~km}$ region of south Taiwan. The highest station elevation is $1792 \mathrm{~m}$ a.s.l. at C1V270, and the lowest station elevation is $105 \mathrm{~m}$ a.s.l. at C10830. The standard deviation of station elevation is $561 \mathrm{~m}$. The values of standard deviation of daily rainfall at the 19 stations were calculated, and less than 13\% except a high standard deviation, $45 \%$, on sixth August (average daily rainfall less than $2 \mathrm{~mm}$ ). The results demonstrated that high and even extreme rainfall are less influenced by elevation, while low and medium rainfall events are significantly influenced by elevation variation, with most of the rainfall appearing on high elevations. Similar results have also been reported by some previous studies (Sanchez-Moreno et al., 2014; Ge et al., 2017). Because the study only considered the rainfall events with total cumulated rainfall greater than $500 \mathrm{~m}$, the elevation effect was ignored as selecting rain station. 


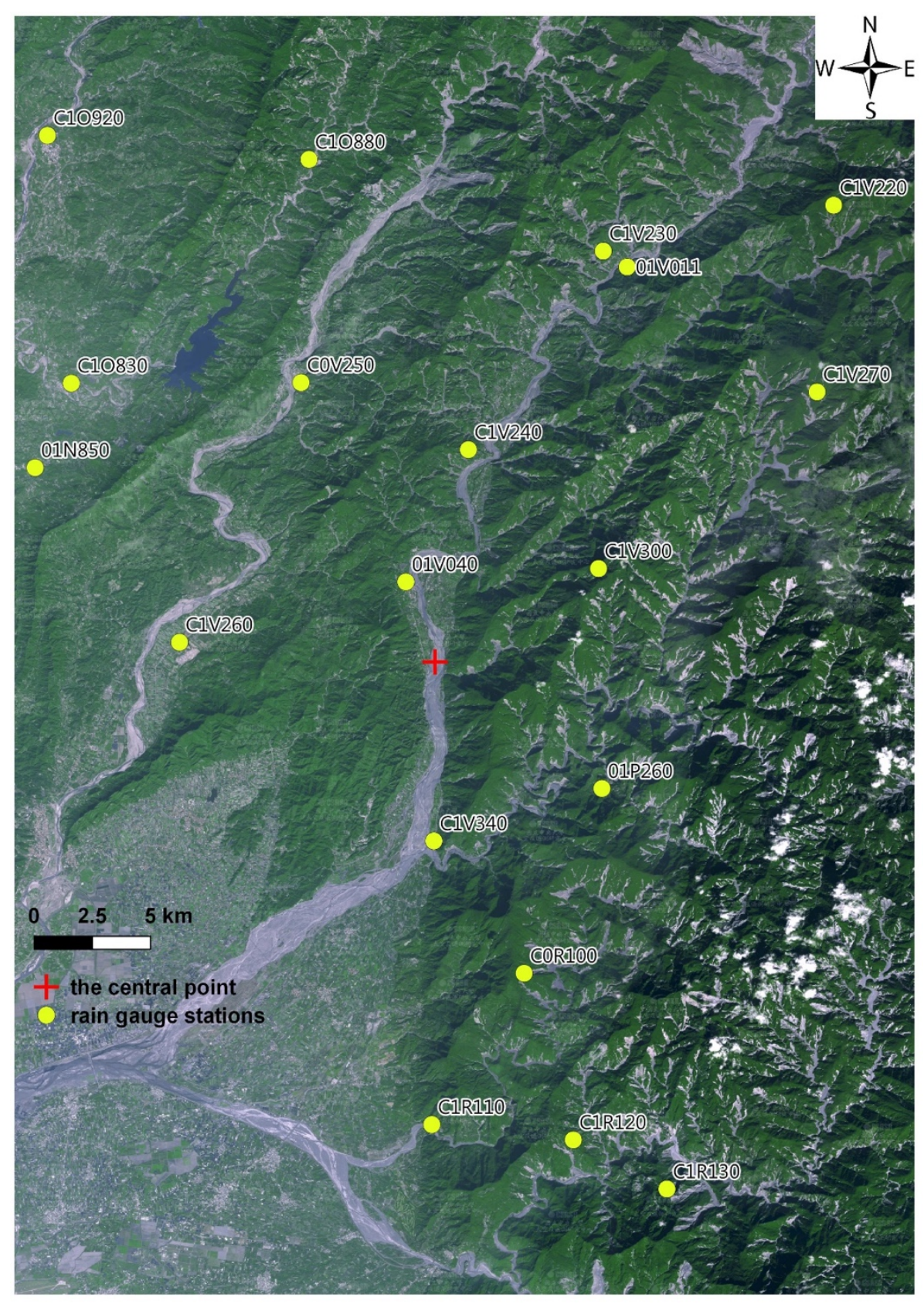

Fig. S2. The distribution of rain gauge stations and the location of the central point of the testing area for validating the influence of the distance between rain gauge and a given point. 


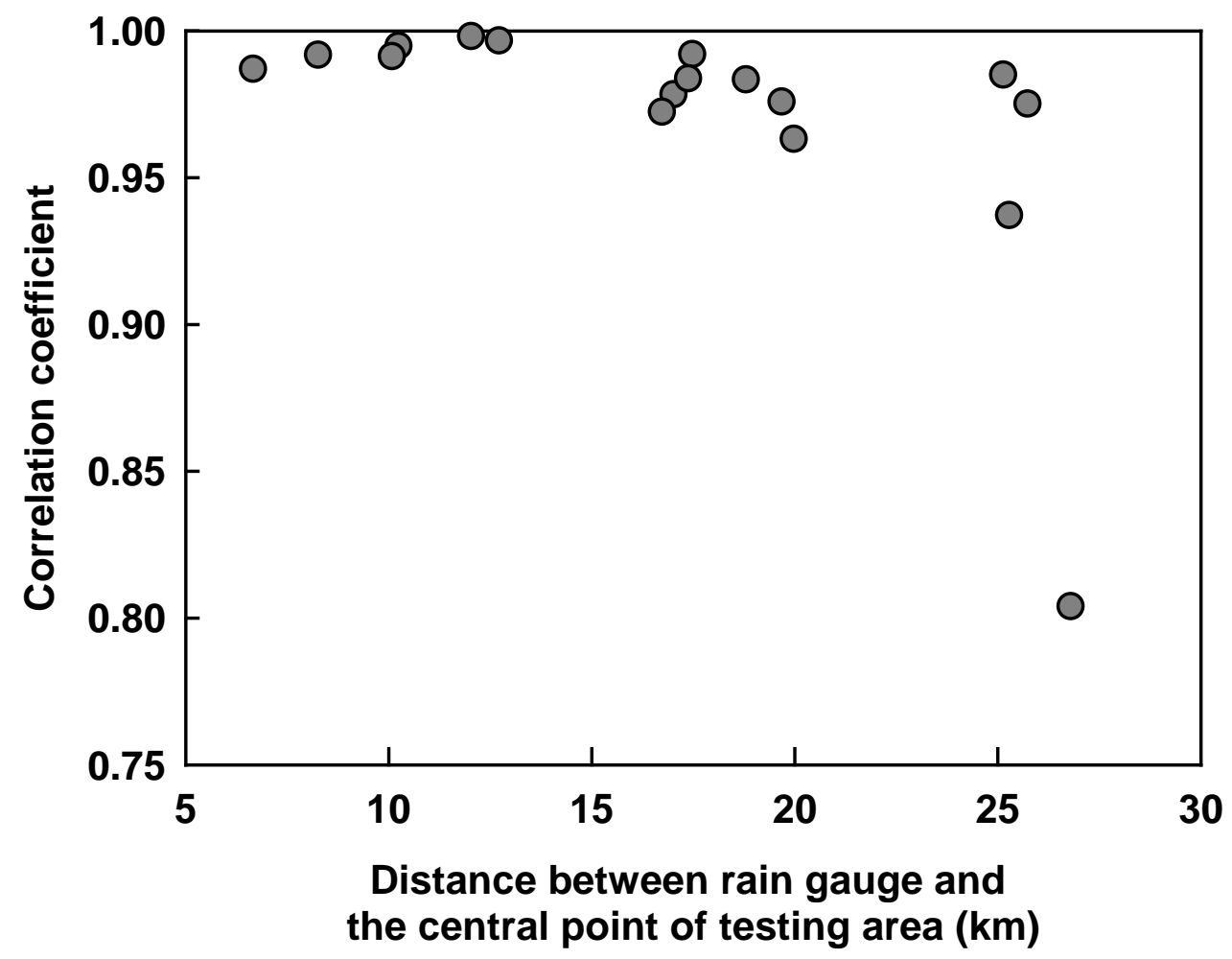

Fig. S3. Variation of correlation coefficient

\section{Reference}

Mishra, A.K. (2013) Effect of rain gauge density over the accuracy of rainfall: a case study over Bangalore, India. SpringerPlus, 2, 311.

Sanchez-Moreno, J.F., Mannaerts, C.M., and Jetten, V. (2014) Influence of topography on rainfall variability in Santiago Island, Cape Verde. International Journal of Climatology, 34, 1081-1097.

Ge, G., Shi, Z., Yang, X., Hao, Y., Guo, H., Kossi, F., Xin, Z., Wei, W., Zhang, Z., Zhang, X., Liu, Y., and Liu, J. (2017) Analysis of Precipitation Extremes in the Qinghai-Tibetan Plateau, China: Spatio-Temporal Characteristics and Topography Effects. Atmosphere, 8(7), 127, doi:10.3390/atmos8070127. 


\section{S4. Detailed information on the 62 detected large landslides}

Table S2. List of the 62 detected large landslides

\begin{tabular}{|c|c|c|c|c|c|c|c|c|c|c|}
\hline ID & $\begin{array}{c}\text { Date and time } \\
\text { (UTC) }\end{array}$ & Longitude & Latitude & $\begin{array}{l}\text { Disturbed } \\
\text { area }\left(\mathrm{km}^{2}\right)\end{array}$ & $\begin{array}{c}\text { Elev. of } \\
\text { landslide (m) }\end{array}$ & $\begin{array}{l}\text { Rain } \\
\text { station }\end{array}$ & $\begin{array}{c}\text { Distance } \\
(\mathrm{km})\end{array}$ & $\begin{array}{c}\text { Elev. of } \\
\text { rain station } \\
\text { (m a.s.l.) }\end{array}$ & $\begin{array}{l}\text { Reactive } \\
\text { landslide } \\
\text { ( } \mathrm{R} \text { or } \mathrm{N} \text { ) }\end{array}$ & $\begin{array}{c}\text { Date of } \\
\text { satellite image }\end{array}$ \\
\hline 1 & 2005/07/18 19:42 & 120.74 & 22.80 & 0.13 & 1388.6 & C1R120 & 5.3 & 820 & $\mathrm{~N}$ & $2005 / 07 / 25$ \\
\hline 2 & 2005/07/20 18:15 & 120.75 & 22.74 & 0.13 & 813.7 & C1R130 & 0.9 & 1040 & $\mathrm{~N}$ & $2005 / 07 / 25$ \\
\hline 3 & 2005/07/20 21:55 & 120.82 & 22.88 & 0.12 & 1535.0 & 01P260 & 10.8 & 458 & $\mathrm{~N}$ & $2005 / 07 / 25$ \\
\hline 4 & 2005/07/21 06:33 & 120.72 & 22.85 & 0.18 & 950.1 & C0R100 & 3.9 & 1006 & $\mathrm{R}$ & 2006/07/29 \\
\hline 5 & 2006/06/09 16:53 & 121.33 & 24.29 & 0.11 & 2304.6 & C1H860 & 24.1 & 1840 & $\mathrm{R}$ & 2006/07/19 \\
\hline 6 & 2008/07/18 21:30 & 121.01 & 23.82 & 0.10 & 1093.8 & C1I040 & 1.6 & 1693 & $\mathrm{R}$ & $2008 / 11 / 20$ \\
\hline 7 & 2008/07/18 23:55 & 120.66 & 23.15 & 0.12 & 749.0 & C1V230 & 6.0 & 760 & $\mathrm{~N}$ & $2008 / 11 / 25$ \\
\hline 8 & 2008/09/15 02:45 & 121.38 & 24.35 & 0.14 & 2236.4 & 41U090 & 5.7 & 1930 & $\mathrm{R}$ & 2008/12/03 \\
\hline 9 & 2008/09/17 18:50 & 121.00 & 24.10 & 0.89 & 1104.3 & $01 \mathrm{~F} 100$ & 2.6 & 1600 & $\mathrm{~N}$ & 2008/11/15 \\
\hline 10 & 2009/08/08 00:04 & 120.72 & 22.57 & 0.39 & 1207.1 & C1R240 & 10.9 & 74 & $\mathrm{R}$ & $2010 / 04 / 10$ \\
\hline 11 & 2009/08/08 00:35 & 120.73 & 22.49 & 0.12 & 950.2 & 01Q350 & 6.2 & 700 & $\mathrm{~N}$ & $2010 / 04 / 10$ \\
\hline 12 & 2009/08/08 01:20 & 120.77 & 23.49 & 0.14 & 1411.2 & H1M240 & 5.2 & 1850 & $\mathrm{~N}$ & $2010 / 02 / 23$ \\
\hline 13 & 2009/08/08 02:20 & 120.85 & 22.98 & 0.11 & 2167.4 & 01P260 & 15.2 & 458 & $\mathrm{R}$ & $2010 / 04 / 10$ \\
\hline 14 & 2009/08/08 03:55 & 120.75 & 23.08 & 0.33 & 923.5 & C1V270 & 5.4 & 1792 & $\mathrm{R}$ & $2010 / 02 / 23$ \\
\hline 15 & 2009/08/08 05:35 & 120.83 & 23.52 & 0.50 & 1903.4 & СОН9АО & 2.4 & 1595 & $\mathrm{~N}$ & $2010 / 02 / 23$ \\
\hline 16 & 2009/08/08 06:25 & 120.82 & 23.06 & 0.39 & 1726.3 & 01V040 & 20.8 & 265 & $\mathrm{~N}$ & $2010 / 02 / 23$ \\
\hline
\end{tabular}




\begin{tabular}{|c|c|c|c|c|c|c|c|c|c|c|}
\hline ID & $\begin{array}{c}\text { Date and time } \\
\text { (UTC) }\end{array}$ & Longitude & Latitude & $\begin{array}{l}\text { Disturbed } \\
\text { area }\left(\mathrm{km}^{2}\right)\end{array}$ & $\begin{array}{c}\text { Elev. of } \\
\text { landslide (m) }\end{array}$ & $\begin{array}{l}\text { Rain } \\
\text { station }\end{array}$ & $\begin{array}{c}\text { Distance } \\
(\mathrm{km})\end{array}$ & $\begin{array}{c}\text { Elev. of } \\
\text { rain station } \\
\text { (m a.s.l.) }\end{array}$ & $\begin{array}{l}\text { Reactive } \\
\text { landslide } \\
\text { ( } \mathrm{R} \text { or } \mathrm{N} \text { ) }\end{array}$ & $\begin{array}{c}\text { Date of } \\
\text { satellite image }\end{array}$ \\
\hline 17 & 2009/08/08 06:28 & 120.67 & 23.01 & 0.15 & 517.2 & 01V040 & 4.0 & 265 & $\mathrm{~N}$ & $2010 / 02 / 23$ \\
\hline 18 & 2009/08/08 07:15 & 120.70 & 23.01 & 0.23 & 903.9 & C1V300 & 1.5 & 1637 & $\mathrm{~N}$ & 2010/02/23 \\
\hline 19 & 2009/08/08 07:35 & 120.70 & 22.75 & 0.49 & 647.8 & C1R120 & 1.0 & 820 & $\mathrm{R}$ & 2010/02/23 \\
\hline 20 & 2009/08/08 08:10 & 120.81 & 23.00 & 0.19 & 2007.5 & C1V300 & 10.1 & 1637 & $\mathrm{R}$ & $2010 / 02 / 23$ \\
\hline 21 & 2009/08/08 08:20 & 120.91 & 23.33 & 0.41 & 1703.8 & 01V070 & 6.3 & 2230 & $\mathrm{~N}$ & 2010/01/11 \\
\hline 22 & 2009/08/08 09:01 & 120.79 & 22.61 & 0.62 & 1222.0 & $01 \mathrm{Q} 910$ & 13.4 & 1158 & $\mathrm{R}$ & $2010 / 04 / 10$ \\
\hline 23 & 2009/08/08 10:40 & 120.86 & 22.80 & 0.16 & 1424.2 & $01 \mathrm{Q} 910$ & 12.8 & 1158 & $\mathrm{R}$ & 2010/04/10 \\
\hline 24 & 2009/08/08 11:35 & 120.95 & 23.33 & 0.22 & 2029.6 & C1V170 & 15.1 & 3340 & $\mathrm{~N}$ & $2010 / 01 / 11$ \\
\hline 25 & 2009/08/08 13:56 & 120.66 & 22.96 & 0.11 & 407.3 & 01V040 & 5.0 & 265 & $\mathrm{~N}$ & 2010/02/23 \\
\hline 26 & 2009/08/08 16:15 & 120.88 & 23.18 & 0.14 & 2162.1 & C1V220 & 7.4 & 1781 & $\mathrm{R}$ & $2010 / 01 / 11$ \\
\hline 27 & 2009/08/08 17:05 & 120.71 & 22.49 & 0.94 & 1168.5 & C1R240 & 9.3 & 74 & $\mathrm{~N}$ & $2010 / 04 / 10$ \\
\hline 28 & 2009/08/08 17:21 & 120.90 & 23.07 & 0.28 & 2606.3 & C1V270 & 9.8 & 1792 & $\mathrm{R}$ & $2010 / 04 / 10$ \\
\hline 29 & 2009/08/08 17:53 & 120.91 & 23.08 & 0.19 & 2459.5 & C1V270 & 10.7 & 1792 & $\mathrm{R}$ & $2010 / 04 / 10$ \\
\hline 30 & 2009/08/08 18:11 & 120.79 & 23.51 & 1.12 & 1763.8 & 467530 & 2.8 & 2413.4 & $\mathrm{~N}$ & $2010 / 02 / 23$ \\
\hline 31 & 2009/08/08 18:16 & 120.83 & 22.63 & 0.72 & 1055.7 & $01 \mathrm{Q} 910$ & 13.5 & 1158 & $\mathrm{R}$ & 2010/04/10 \\
\hline 32 & 2009/08/08 18:19 & 120.72 & 22.70 & 0.56 & 603.4 & $01 \mathrm{Q} 910$ & 5.3 & 1158 & $\mathrm{R}$ & 2010/03/06 \\
\hline 33 & 2009/08/08 18:28 & 120.66 & 22.95 & 0.12 & 554.4 & 01V040 & 5.8 & 265 & $\mathrm{R}$ & $2010 / 02 / 23$ \\
\hline 34 & 2009/08/08 19:19 & 120.71 & 22.67 & 0.64 & 705.7 & $01 \mathrm{Q} 910$ & 7.6 & 1158 & $\mathrm{R}$ & 2010/03/06 \\
\hline 35 & 2009/08/08 20:15 & 120.73 & 22.59 & 0.73 & 1509.7 & C1R240 & 12.8 & 74 & $\mathrm{~N}$ & 2010/04/10 \\
\hline
\end{tabular}




\begin{tabular}{|c|c|c|c|c|c|c|c|c|c|c|}
\hline ID & $\begin{array}{c}\text { Date and time } \\
\text { (UTC) }\end{array}$ & Longitude & Latitude & $\begin{array}{l}\text { Disturbed } \\
\text { area }\left(\mathrm{km}^{2}\right)\end{array}$ & $\begin{array}{c}\text { Elev. of } \\
\text { landslide (m) }\end{array}$ & $\begin{array}{c}\text { Rain } \\
\text { station }\end{array}$ & $\begin{array}{c}\text { Distance } \\
(\mathrm{km})\end{array}$ & $\begin{array}{c}\text { Elev. of } \\
\text { rain station } \\
\text { (m a.s.l.) }\end{array}$ & $\begin{array}{l}\text { Reactive } \\
\text { landslide } \\
\text { ( } \mathrm{R} \text { or } \mathrm{N} \text { ) }\end{array}$ & $\begin{array}{c}\text { Date of } \\
\text { satellite image }\end{array}$ \\
\hline 36 & 2009/08/08 20:27 & 120.92 & 23.40 & 0.12 & 2278.6 & C1V460 & 4.5 & 1949 & $\mathrm{~N}$ & $2010 / 01 / 11$ \\
\hline 37 & 2009/08/08 21:11 & 120.90 & 23.46 & 0.15 & 1904.4 & C1V460 & 2.3 & 1949 & $\mathrm{R}$ & $2010 / 02 / 23$ \\
\hline 38 & 2009/08/08 21:30 & 120.92 & 23.49 & 0.12 & 2450.4 & C1V460 & 6.4 & 1949 & $\mathrm{~N}$ & $2010 / 02 / 23$ \\
\hline 39 & 2009/08/08 21:42 & 120.91 & 23.10 & 0.25 & 2274.1 & C1V460 & 37.3 & 1949 & $\mathrm{R}$ & $2010 / 04 / 10$ \\
\hline 40 & 2009/08/08 22:16 & 120.66 & 23.17 & 2.50 & 681.3 & C1R880 & 6.4 & 223 & $\mathrm{R}$ & $2010 / 02 / 23$ \\
\hline 41 & 2009/08/08 22:52 & 120.90 & 23.54 & 0.12 & 1936.8 & C1I340 & 4.5 & 897 & $\mathrm{R}$ & $2010 / 02 / 23$ \\
\hline 42 & 2009/08/08 23:02 & 120.60 & 23.03 & 0.13 & 747.9 & C0V250 & 5.2 & 298 & $\mathrm{~N}$ & $2010 / 04 / 10$ \\
\hline 43 & 2009/08/08 23:14 & 120.75 & 23.29 & 0.56 & 1525.1 & C1V200 & 7.7 & 860 & $\mathrm{R}$ & $2010 / 02 / 23$ \\
\hline 44 & 2009/08/08 23:15 & 120.77 & 22.63 & 0.15 & 2309.0 & 01Q250 & 8.2 & 950 & $\mathrm{R}$ & $2010 / 04 / 10$ \\
\hline 45 & 2009/08/08 23:41 & 120.84 & 22.63 & 0.12 & 825.0 & $01 Q 910$ & 13.9 & 1158 & $\mathrm{R}$ & $2010 / 04 / 10$ \\
\hline 46 & 2009/08/09 00:34 & 120.77 & 23.22 & 2.24 & 1352.5 & C1V210 & 4.0 & 700 & $\mathrm{R}$ & $2010 / 02 / 23$ \\
\hline 47 & 2009/08/09 02:52 & 120.77 & 23.23 & 0.81 & 1559.3 & C1V210 & 4.1 & 700 & $\mathrm{R}$ & $2010 / 02 / 23$ \\
\hline 48 & 2009/08/09 03:55 & 120.72 & 22.60 & 0.63 & 923.5 & 01Q250 & 2.5 & 950 & $\mathrm{~N}$ & $2010 / 04 / 10$ \\
\hline 49 & 2009/08/09 09:37 & 120.81 & 22.56 & 2.31 & 1144.3 & 01Q350 & 14.3 & 250 & $\mathrm{~N}$ & $2010 / 04 / 10$ \\
\hline 50 & 2009/08/09 11:00 & 120.77 & 22.82 & 0.13 & 1669.4 & C1R120 & 9.0 & 820 & $\mathrm{R}$ & $2010 / 04 / 10$ \\
\hline 51 & 2009/08/10 03:54 & 120.80 & 23.25 & 0.20 & 1227.6 & C1V210 & 2.8 & 700 & $\mathrm{R}$ & $2010 / 02 / 23$ \\
\hline 52 & 2009/08/10 04:22 & 120.76 & 23.31 & 1.52 & 1387.2 & C1V160 & 6.3 & 1040 & $\mathrm{R}$ & $2010 / 02 / 23$ \\
\hline 53 & 2010/09/19 23:24 & 120.73 & 22.85 & 0.15 & 1135.0 & $01 Q 910$ & 13.9 & 1158 & $\mathrm{R}$ & $2011 / 04 / 16$ \\
\hline 54 & 2011/08/30 07:10 & 120.93 & 22.86 & 0.12 & 849.8 & 01Q350 & 44.9 & 1275 & $\mathrm{R}$ & $2012 / 02 / 27$ \\
\hline
\end{tabular}




\begin{tabular}{|c|c|c|c|c|c|c|c|c|c|c|}
\hline ID & $\begin{array}{c}\text { Date and time } \\
\text { (UTC) }\end{array}$ & Longitude & Latitude & $\begin{array}{l}\text { Disturbed } \\
\text { area }\left(\mathrm{km}^{2}\right)\end{array}$ & $\begin{array}{c}\text { Elev. of } \\
\text { landslide (m) }\end{array}$ & $\begin{array}{c}\text { Rain } \\
\text { station }\end{array}$ & $\begin{array}{c}\text { Distance } \\
(\mathrm{km})\end{array}$ & $\begin{array}{c}\text { Elev. of } \\
\text { rain station } \\
\text { (m a.s.l.) }\end{array}$ & $\begin{array}{l}\text { Reactive } \\
\text { landslide } \\
\text { (R or N) }\end{array}$ & $\begin{array}{c}\text { Date of } \\
\text { satellite image }\end{array}$ \\
\hline 55 & 2011/08/30 09:13 & 121.18 & 23.69 & 0.11 & 1811.5 & C1T940 & 19.6 & 1570 & $\mathrm{R}$ & $2012 / 02 / 27$ \\
\hline 56 & 2011/08/31 09:37 & 120.98 & 23.33 & 0.11 & 2714.0 & 01V070 & 8.5 & 2230 & $\mathrm{R}$ & $2012 / 02 / 27$ \\
\hline 57 & 2012/08/01 18:40 & 121.42 & 24.58 & 0.12 & 1512.0 & 01U050 & 8.1 & 400 & $\mathrm{R}$ & 2013/07/11 \\
\hline 58 & 2012/08/02 10:00 & 121.85 & 24.52 & 0.12 & 83.3 & C0U710 & 33.3 & 1810 & $\mathrm{~N}$ & 2013/06/28 \\
\hline 59 & 2012/08/02 19:00 & 120.95 & 23.74 & 0.25 & 1677.9 & C1I310 & 6.6 & 1001 & $\mathrm{~N}$ & 2013/06/03 \\
\hline 60 & 2012/08/03 01:02 & 121.38 & 24.36 & 0.19 & 2356.6 & 41U090 & 4.7 & 1930 & $\mathrm{~N}$ & 2013/07/11 \\
\hline 61 & 2013/07/13 14:27 & 120.89 & 23.02 & 0.40 & 2604.8 & C1V270 & 10.1 & 1792 & $\mathrm{R}$ & $2014 / 07 / 13$ \\
\hline 62 & 2013/08/22 19:05 & 121.07 & 23.38 & 0.18 & 2114.6 & C1I140 & 41.2 & 1700 & $\mathrm{R}$ & 2014/07/13 \\
\hline
\end{tabular}

The average location error (the distance between the actual and estimated location) was $10.9 \mathrm{~km}$. The best location estimate was for the ID 40 landslide with an error of $0.5 \mathrm{~km}$, while the worst location estimate was for ID 35 landslide with an error of $49.3 \mathrm{~km}$. 
S5. Physically-based I-D thresholds reported by the previous studies

Table S3. Three physically-based I-D thresholds reported by the previous studies

\begin{tabular}{llll}
\hline & Reference & Equation & Study area \\
\hline 1 & Salciarini et al. (2012) & $I=276.2 D^{-0.99}$ & Model \\
2 & Chen et al. (2013c) & $I=24.4 D^{-0.28}$ & Taiwan \\
3 & Napolitano et al. (2016) & $I=287.8 D^{-1.09}$ & southern Italy \\
\hline
\end{tabular}

